\title{
Angiotensin-Converting Enzyme Inhibitor Therapy Effects in Patients With Heart Failure With Preserved and Mid-Range Ejection Fraction
}

\author{
Julia Safonova ${ }^{a}$ b (D), Maria Kozhevnikova ${ }^{a}$, Yulia Danilogorskaya ${ }^{a}$, Elena Zheleznykha, \\ Vita Zektser ${ }^{\mathrm{a}}$, Irina Ilgisonis ${ }^{\mathrm{a}}$, Lyudmila Popova ${ }^{\mathrm{a}}$, Natalia Khabarova ${ }^{\mathrm{a}}$, \\ Elena Privalova ${ }^{a}$, Yuri Belenkov ${ }^{\mathrm{a}}$
}

\begin{abstract}
Background: There is hypothesis that endothelial function enhancement is strongly associated with better outcome and functional class improvement in heart failure with preserved ejection fraction (HF$\mathrm{pEF}$ ) and heart failure with mid-range ejection fraction (HFmrEF) patients. Perindopril is the only angiotensin-converting enzyme inhibitor (ACEI) drug with proven positive effect on the endothelium in coronary artery disease (CAD) patients. In patients with $\mathrm{HFpEF}$ and HFmrEF, its impact is still unknown. The aim of this study was to assess perindopril's influence on endothelial dysfunction markers in these groups of patients.
\end{abstract}

Methods: We included 60 patients with HFpEF and HFmrEF. At the baseline, endothelial dysfunction biomarkers were measured by IFA and echocardiographic parameters (left atrial volume index (LAVI), ejection fraction (EF), left ventricular mass index (LVMI), left ventricular end-diastolic diameter (LVEDD), and left ventricular enddiastolic volume (LVEDV)) were studied. In patients with no history of previous ACEI or angiotensin II receptor blockers (ARBs) therapy, perindopril was prescribed for 12 months. If patient was treated with ARB or ACEI drug other than perindopril before the study, after 48 -h withdrawal period, previous drug was replaced by perindopril.

Results: After 12-month therapy with perindopril, E-selectin decreased from 57.25 to $46.05 \mathrm{ng} / \mathrm{mL}$ and from 56.55 to $47.6 \mathrm{ng} / \mathrm{mL}$ in HFpEF and HFmrEF patients, respectively $(\mathrm{P}<0.05)$. Significant reductions from 0.99 to $0.76 \mathrm{pg} / \mathrm{mL}(\mathrm{P}<0.05)$ and from 1.08 to 0.97 $\mathrm{pg} / \mathrm{mL}(\mathrm{P}<0.05)$ in endothelin-1 level were shown in patients with HFpEF and HFmrEF.

Conclusion: The 12-month therapy with perindopril leads to LAVI

Manuscript submitted September 13, 2021, accepted October 4, 2021

Published online November 5, 2021

a.M. Sechenov First Moscow State Medical University, Moscow, Russia ${ }^{b}$ Corresponding Author: Julia Safonova, I.M. Sechenov First Moscow State Medical University, Room 106, 2-4 Bolshaya Pirogovskaya Str., Moscow 119991, Russia. Email: thunderbird.3194@gmail.com

doi: https://doi.org/10.14740/cr1322 reduction in HFmrEF patients and potential endothelial dysfunction markers decrease in HFpEF and HFmrEF patients.

Keywords: HF; Angiotensin-converting enzyme inhibitor; HFpEF; $\mathrm{HFmrEF}$

\section{Introduction}

Heart failure (HF) is one of the most important global health issues. Approximately 23 million patients have HF diagnosis [1], and prevalence of patients with HF is expected to increase up to $46 \%$ by 2030 [2]. Up to date, the HF pathogenesis, especially $\mathrm{HF}$ with preserved (HFpEF) and mid-range ejection fraction (HFmrEF), is of great interest. Experimental data showed the key role of endothelial dysfunction in the HFpEF development [3].

Concomitant diseases, such as anemia, chronic obstructive pulmonary disease, obesity and diabetes mellitus contribute to the development of a pro-inflammatory state, synthesis of reactive oxygen species and the development of oxidative stress, which leads to impaired vasomotor function. Along with this, in response to the development of oxidative stress, the E-selectin expression by the endothelium increases [3, 4], what makes E-selectin, along with endothelin-1 (ET-1), a potential marker of endothelial dysfunction, but their contribution to the pathogenesis of HFpEF still remains to be elucidated. Despite the fact that HFmrEF is phenotypically closer to heart failure with reduced ejection fraction (HFrEF) [5], most researchers are inclined to believe that HFmrEF is a transitional stage between HFpEF and HFrEF. Taking into account that E-selectin and ET-1 are often considered as the potential markers of endothelial dysfunction in patients with HF, dynamic level determination of these markers during therapy with perindopril could help in search of target therapy.

\section{Materials and Methods}

We conducted a prospective observational study at the Hospital Therapy No. 1 Clinic I.M. Sechenov First Moscow State 
Medical University. The research protocol was approved by the Institution Review Board. All participants gave written consent prior to enrollment. The study was conducted in compliance with the ethical standards of the responsible institution on human subjects as well as with the Helsinki Declaration.

One hundred patients with HFpEF and HFmrEF treated with angiotensin-converting enzyme inhibitor (ACEI) other than perindopril or angiotensin II receptor blocker (ARB) were assessed for eligibility. Diagnosis was established by presence of HF symptoms, N-terminal prohormone of brain natriuretic peptide (NT-proBNP) level $125 \mathrm{pg} / \mathrm{mL}$ or higher and echocardiographic parameters, according to the European Society of Cardiology (ESC) Acute and Chronic Heart Failure Guidelines (2016).

Exclusion criteria were acute coronary syndrome, valvular heart disease with hemodynamically significant stenosis or insufficiency, confirmed oncological disease which requires polychemotherapy and/or radiation therapy at the time of inclusion in the study, the presence of chronic viral diseases (human immunodeficiency virus (HIV), viral hepatitis B and C), acute infectious diseases, severe liver dysfunction (including liver cirrhosis), acute renal failure, mental illness, pregnancy and lactation. Forty patients were excluded from the study: oncologic diagnosis was verified in 10 patients and soon after that chemotherapy course was started, 20 patients died from coronavirus disease 2019 (COVID-19) and other 10 patients changed perindopril to ARBs because of side-effect (cough). So, only 60 patients were enrolled in this study.

\section{Study design}

Endothelial dysfunction biomarkers and NT-proBNP were collected, echocardiography and 6-min walking test (6-MWT) were performed in group of enrolled patients at the baseline (before perindopril prescription). Patients, who were previously on therapy with the other drugs from the ACEI group or ARBs, after 48-h washout period were prescribed perindopril. Patients who had not previously taken an ACEI or ARB were first prescribed perindopril with dose titration to the maximum tolerated. After 12-month therapy with perindopril biomarker levels, echocardiographic parameters and 6-MWT were redetermined. Life quality was assessed at the baseline and after 12-month therapy with perindopril by means of Minnesota Living with Heart Failure Questionnaire (MLHFQ).

Human serum ET-1 was measured by EnzoLife Scientific (USA), while human serum E-selectin level was measured by Technoclon (Technozym E-selectin: AgEIISE) (Austria). NTproBNP level was determined by Triage Meter Pro NT-proBNP test (Biosite, USA).

\section{Statistical analysis}

Statistical indicators such as median and interquartile range were used to present quantitative data, while absolute values and percentage were used for qualitative data assessment. Differences in two dependent variables were assessed by Wilcoxon matched pairs test. To establish difference between two independent groups, Mann-Whitney U test was used. Categorical values were compared with the Chi-square test. All results were considered statistically significant at $\mathrm{P}<0.05$. Statistical analysis was performed using STATISTICA 12.0 (StatSoft Inc., USA).

\section{Results}

\section{Baseline characteristics}

Sixty patients with symptomatic New York Heart Association (NYHA) II-III functional class (FC) HFpEF $(\mathrm{n}=30)$ and HFm$\mathrm{rEF}(\mathrm{n}=30)$ were enrolled in this study. The average age was 66.5 years in HFpEF and 68 in HFmrEF group. There was no difference in age, sex and medication use. All patients had pre-obesity or obesity and hypertension, while $95 \%$ and $90 \%$ of patients in $\mathrm{HFpEF}$ and HFmrEF groups had CAD. All patients were treated with beta-blockers and mineralocorticoid receptor antagonists. High levels of NT-proBNP and E-selectin were identified. At the same time, ET-1 stayed within normal range (Table 1).

\section{Cardiovascular parameters during perindopril therapy}

Blood pressure decreased in HFpEF and HFmrEF patients: from $152 / 94$ to $134 / 80 \mathrm{~mm} \mathrm{Hg}$ in $\mathrm{HFpEF}$ group and from 154/90 to $134 / 80 \mathrm{~mm} \mathrm{Hg}$ in HFmrEF group ( $\mathrm{P}<0.05$ for both groups). Slight, but significant decrease in heart rate from 69 to $66 \mathrm{bpm}(\mathrm{P}$ $=0.001)$ and from 68 to $63 \mathrm{bpm}(\mathrm{P}=0.001)$ was observed in HFpEF and HFmrEF groups, respectively. Also 6-MWT distance and life quality, based on MLHFQ, improved on the therapy with perindopril. According to the 6-MWT, distance increased from 429 to $452 \mathrm{~m}$ in $\mathrm{HFpEF}$ and from 407 to $427 \mathrm{~m}$ in patients with HFmrEF ( $\mathrm{P}<0.05$ for both groups). MLHFQ improved from 29.5 to 18.5 points in patients with $\mathrm{HFpEF}$ and from 37.5 to 23.5 points in patients with HFmrEF ( $\mathrm{P}<0.05$ for both groups).

\section{Cardiac remodeling in HFpEF and HFmrEF patients treated with perindopril for 12 months}

After 12-month therapy with perindopril, slight reduction in left atrial volume index (LAVI) was found in patients with HFmrEF, but it did not reach statistical significance $(P=0.1)$. Left ventricular mass index (LVMI) slightly decreased in HF$\mathrm{pEF}$ group of patients from 115 to $114.8 \mathrm{~mL} / \mathrm{m}^{2}(\mathrm{P}=0.04)$. Ejection fraction improved only in HFmrEF patients $(\mathrm{P}=$ $0.0002)$, whereas ejection fraction did not change in HFpEF group $(\mathrm{P}=0.9)$ (Table 2). Eight out of 30 patients with HFm$\mathrm{rEF}$ shifted to HFpEF group on basis of ejection fraction improvement after 12-month period of therapy with perindopril.

\section{Biomarker levels dynamics on therapy with perindopril}

The reduction in E-selectin and ET-1 levels was shown in HFpEF group of patients. E-selectin markedly declined from $57.25(44.9-74.1)$ to $46.05 \mathrm{ng} / \mathrm{mL}(37.8$ - 66.2) $(\mathrm{P}=0.000002)$, 
Table 1. Baseline Characteristics

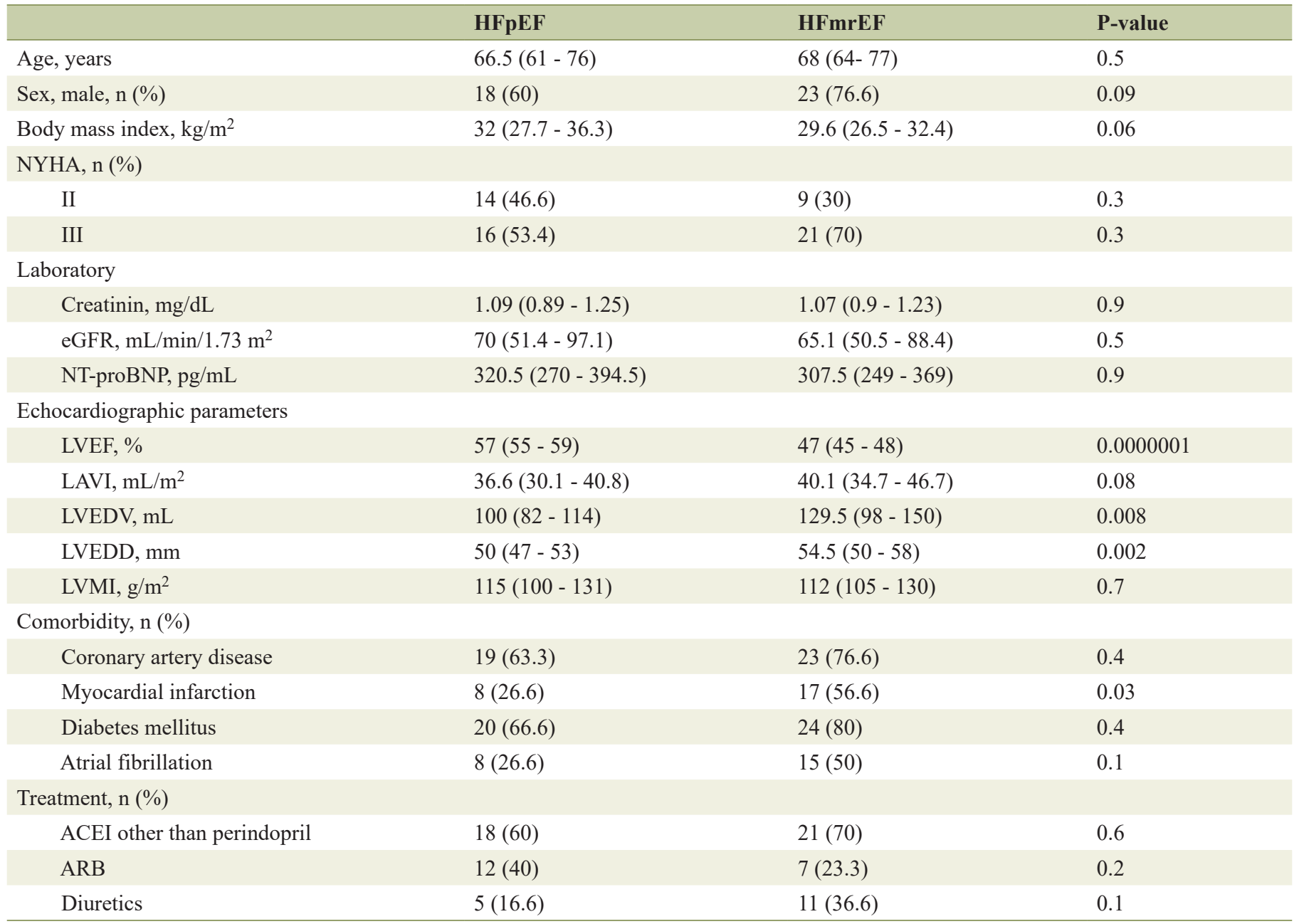

HFpEF: heart failure with preserved ejection fraction; HFmrEF: heart failure with mid-range ejection fraction; NYHA: New York Heart Association; eGFR: estimated glomerular filtration rate; NT-proBNP: N-terminal prohormone of brain natriuretic peptide; LVEF: left ventricular ejection fraction; LAVI: left atrial volume index; LVEDV: left ventricular end-diastolic volume; LVEDD: left ventricular end-diastolic diameter; LVMI: left ventricular mass index; ACEl: angiotensin-converting enzyme; ARB: angiotensin II receptor blocker.

whereas ET-1 decreased from $0.99(0.54-1.52)$ to $0.76 \mathrm{pg} / \mathrm{mL}$ (0.48 - 1.28) $(\mathrm{P}=0.00003)$.

In HFmrEF patients, E-selectin level decreased from 56.55 (34 - 64.2) to $47.6 \mathrm{ng} / \mathrm{mL}(30.2-54.3)(\mathrm{P}=0.0003)$ after 12 -month therapy with perindopril. At the same time, ET-1 level declined from $1.08(0.79-1.48)$ to $0.97 \mathrm{pg} / \mathrm{mL}(0.65-1.22)(\mathrm{P}=0.02)$.

Regarding NT-proBNP, the same results were demonstrated. Significant reduction in its level was shown in both groups of patients: NT-proBNP decreased from 320.5 (270 $394.5)$ to $258 \mathrm{pg} / \mathrm{mL}(197.5$ - 325.5) in patients with HFpEF (P $=0.00008)$, whereas in HFmrEF, its level declined from 307.5 (249 - 369) to $243.5(196.5$ - 291.5) $(\mathrm{P}=0.00008)$ (Table 3).

\section{Discussion}

Our study demonstrated that E-selectin and ET-1 level decrease after 12-month therapy with perindopril in patients with HF-
pEF and HFmrEF. As derivates of endothelium, E-selectin and ET-1 might be considered as endothelial dysfunction markers. Since endothelial dysfunction is the cornerstone of HFpEF and HFmrEF, we suppose that perindopril can positively affect endothelial function and lead to ejection fraction improvement and better outcome in HFpEF and HFmrEF patients.

The recent data support a suggestion that endothelial dysfunction improvement is the key to HFpEF and HFmrEF management. It is thought that an improved endothelial function can lead to better prognosis and functional status improvement in these groups of patients [6].

Up to date, the relationship of markers of endothelial dysfunction (E-selectin and ET-1) with the activation of the reninangiotensin-aldosterone system (RAAS) was shown [3, 7, 8]. RAAS is one of the many mechanisms that take part in the systemic inflammation development. RAAS induces an increase in the synthesis of reactive oxygen species and adhesion molecules, which in turn leads to impaired endothelial function [9]. 
Table 2. Echocardiographic Parameters Before and After 12-Month Therapy With Perindopril

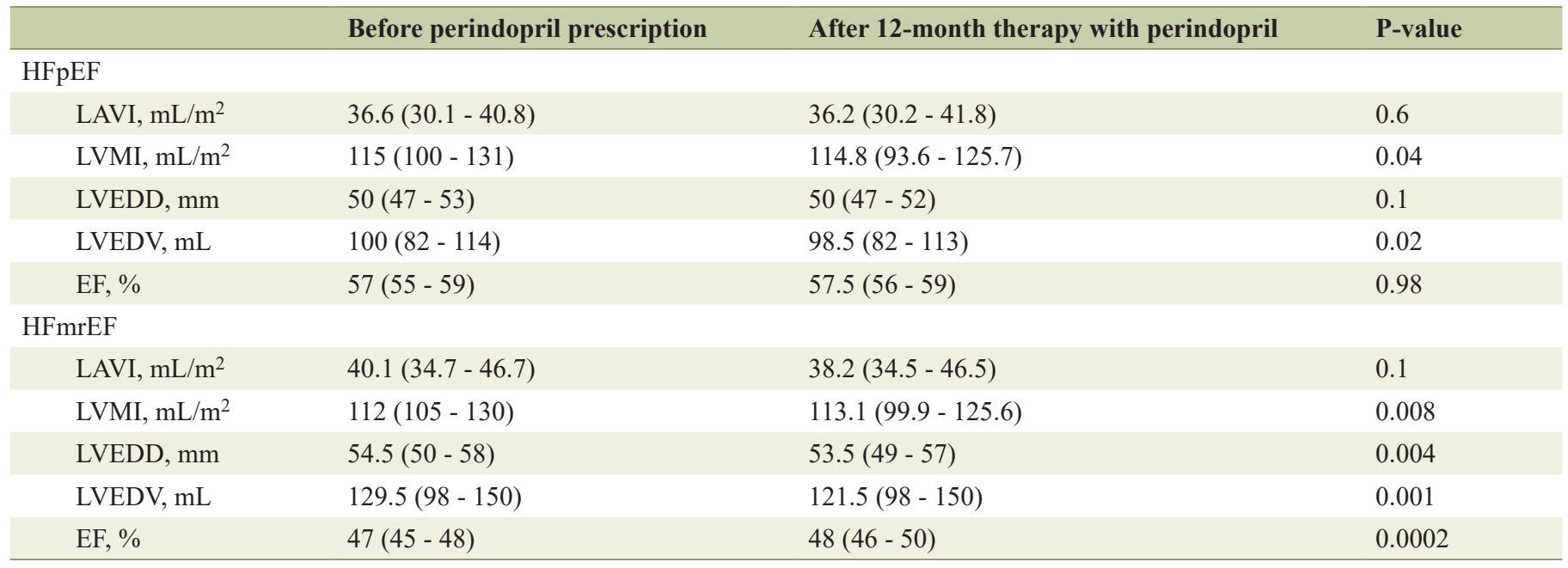

HFpEF: heart failure with preserved ejection fraction; HFmrEF: heart failure with mid-range ejection fraction; LAVI: left atrial volume index; LVMI: left ventricular mass index; LVEDD: left ventricular end-diastolic diameter; LVEDV: left ventricular end-diastolic volume; EF: ejection fraction.

It can be assumed that inhibition of the RAAS, on the contrary, will lead to an improvement of endothelial function in patients with HFpEF and HFmrEF, since their pathogenesis is based on endothelial dysfunction as a result of the systemic inflammation development. Experimental data indicate that ACEIs are able to reduce the level of ET-1 [7]. But ACEI therapy's influence on E-selectin level is still unknown.

Perindopril is an ACEI drug, which realizes its effect by suppressing the activity of the RAAS. In comparison with the other ACEIs, perindopril has proven effect on endothelial function [10-13]. The perindopril prescription in patients with HFpEF and HFmrEF remains controversial. Currently, there is no evidence base for its use in these groups of patients. However, in the presence of such diseases as hypertension and CAD in patients with $\mathrm{HFpEF}$ and HFmrEF, the use of perindopril seems reasonable.

\section{Limitations}

Limitations of this study are small number of patients and absence of instrumental verification of endothelial dysfunction. However, the majority of previous studies, investigating features of HFmrEF, include number of patients less than 70 [14-19], what can be explained by lower prevalence of this phenotype. Also, no control group was provided, but previous studies showed higher levels of E-selectin and abnormal structural and functional parameters of microcirculatory vessels in patients with cardiovascular diseases in comparison to healthy patients [20, 21]. Despite limitations, this pilot study data appear to be promising and pathogenetically valid in patients with HFpEF and HFmrEF.

\section{Acknowledgments}

We acknowledge RFBR for funding the research.

\section{Financial Disclosure}

The reported study was funded by Russian Foundation for Ba-

Table 3. Endothelial Dysfunction and Myocardial Stretch Markers Before and After 12-Month Therapy With Perindopril

\begin{tabular}{|c|c|c|c|}
\hline & Before perindopril prescription & After 12-month therapy with perindopril & P-value \\
\hline \multicolumn{4}{|l|}{ HFpEF } \\
\hline E-selectin, ng/mL & $57.25(44.9-74.1)$ & $46.05(37.8-66.2)$ & 0.000002 \\
\hline NT-proBNP, pg/mL & $320.5(270-394.5)$ & $258(197.5-325.5)$ & 0.00008 \\
\hline \multicolumn{4}{|l|}{ HFmrEF } \\
\hline NT-proBNP, pg/mL & $307.5(249-369)$ & $243.5(196.5-291.5)$ & 0.00008 \\
\hline
\end{tabular}

HFpEF: heart failure with preserved ejection fraction; HFmrEF: heart failure with mid-range ejection fraction; ET-1: endothelin-1; NT-proBNP: Nterminal prohormone of brain natriuretic peptide. 
sic Research (RFBR) according to the research project no. 20315-90078.

\section{Conflict of Interest}

We declare that there is no conflict of interest.

\section{Informed Consent}

Patients underwent informed consented procedures with Sechenov University research staff.

\section{Author Contributions}

Julia Safonova designed the study, collected data, reviewed the literature, analyzed and interpreted the data, and drafted the manuscript. Maria Kozhevnikova engaged in data collection, data interpretation, and contributed to drafts of the manuscript. Yulia Danilogorskaya, Elena Zheleznykh, Vita Zektser, Lyudmila Popova, and Natalia Khabarova engaged in data collection. Irina Ilgisonis contributed to the data collection and provided critical reviews of the manuscript. Elena Privalova contributed to the study design and provided critical reviews of the manuscript. Yuri Belenkov contributed to the design of the study, literature review, data interpretation, and provided critical reviews of the manuscript.

\section{Data Availability}

The authors declare that data supporting the findings of this study are available within the article.

\section{Abbreviations}

ACEI: angiotensin-converting enzyme inhibitor; ARB: angiotensin II receptor blocker; CAD: coronary artery disease; EF: ejection fraction; ET-1: endothelin-1; FC: functional class; HF: heart failure; HFmrEF: heart failure with mid-range ejection fraction; HFpEF: heart failure with preserved ejection fraction; HIV: human immunodeficiency virus; LAVI: left atrial volume index; LVEDD: left ventricular end-diastolic diameter; LVEDV: left ventricular end-diastolic volume; LVMI: left ventricular mass index; RAAS: renin-angiotensin-aldosterone system

\section{References}

1. Ambrosy AP, Fonarow GC, Butler J, Chioncel O, Greene SJ, Vaduganathan M, Nodari S, et al. The global health and economic burden of hospitalizations for heart failure: lessons learned from hospitalized heart failure registries. J Am Coll Cardiol. 2014;63(12):1123-1133.
2. Benjamin EJ, Muntner P, Alonso A, Bittencourt MS, Callaway CW, Carson AP, Chamberlain AM, et al. Heart disease and stroke statistics-2019 update: a report from the American Heart Association. Circulation. 2019;139(10):e56-e528.

3. Paulus WJ, Tschope C. A novel paradigm for heart failure with preserved ejection fraction: comorbidities drive myocardial dysfunction and remodeling through coronary microvascular endothelial inflammation. J Am Coll Cardiol. 2013;62(4):263-271.

4. Franssen C, Chen S, Unger A, Korkmaz HI, De Keulenaer GW, Tschope C, Leite-Moreira AF, et al. Myocardial microvascular inflammatory endothelial activation in heart failure with preserved ejection fraction. JACC Heart Fail. 2016;4(4):312-324.

5. Nauta JF, Hummel YM, van Melle JP, van der Meer P, Lam CSP, Ponikowski P, Voors AA. What have we learned about heart failure with mid-range ejection fraction one year after its introduction? Eur J Heart Fail. 2017;19(12):1569-1573.

6. Gevaert AB, Lemmens K, Vrints CJ, Van Craenenbroeck EM. Targeting Endothelial Function to Treat Heart Failure with Preserved Ejection Fraction: The Promise of Exercise Training. Oxid Med Cell Longev. 2017;2017:4865756.

7. Bakris GL, Bhandaru S, Akerstrom V, Re RN. ACE inhibitor-mediated attenuation of mesangial cell growth. A role for endothelin. Am J Hypertens. 1994;7(7 Pt 1):583-590.

8. Imai T, Hirata Y, Emori T, Yanagisawa M, Masaki T, Marumo F. Induction of endothelin-1 gene by angiotensin and vasopressin in endothelial cells. Hypertension. 1992;19(6 Pt 2):753-757.

9. Brasier AR, Recinos A, 3rd, Eledrisi MS. Vascular inflammation and the renin-angiotensin system. Arterioscler Thromb Vasc Biol. 2002;22(8):1257-1266.

10. Ghiadoni L, Magagna A, Versari D, Kardasz I, Huang Y, Taddei S, Salvetti A. Different effect of antihypertensive drugs on conduit artery endothelial function. Hypertension. 2003;41(6):1281-1286.

11. Ceconi C, Fox KM, Remme WJ, Simoons ML, Bertrand M, Parrinello G, Kluft C, et al. ACE inhibition with perindopril and endothelial function. Results of a substudy of the EUROPA study: PERTINENT. Cardiovasc Res. 2007;73(1):237-246.

12. Bots ML, Remme WJ, Luscher TF, Fox KM, Bertrand M, Ferrari R, Simoons ML, et al. ACE inhibition and endothelial function: main findings of PERFECT, a sub-study of the EUROPA trial. Cardiovasc Drugs Ther. 2007;21(4):269-279.

13. Danilogorskaya Yu A, Zheleznykh EA, Privalova EV, Shchendrygina AA, Belenkov Yu N, Ilgisonis IS, Tishman MI. Influence of the combination drug Prestans (perindopril A, amlodipine) on morpho-functional parameters of arterial bed in essential hypertension. Russian Journal of Cardiology. 2017;12(152):113-119.

14. Shiga T, Suzuki A, Haruta S, Mori F, Ota Y, Yagi M, Oka $\mathrm{T}$, et al. Clinical characteristics of hospitalized heart failure patients with preserved, mid-range, and reduced ejection fractions in Japan. ESC Heart Fail. 2019;6(3):475486. 
15. Margonato D, Mazzetti S, De Maria R, Gorini M, Iacoviello M, Maggioni AP, Mortara A. Heart failure with mid-range or recovered ejection fraction: differential determinants of transition. Card Fail Rev. 2020;6:e28.

16. Webb J, Draper J, Fovargue L, Sieniewicz B, Gould J, Claridge $\mathrm{S}$, Barton $\mathrm{C}$, et al. Is heart failure with mid range ejection fraction (HFmrEF) a distinct clinical entity or an overlap group? Int J Cardiol Heart Vasc. 2018;21:1-6.

17. Kuzheleva EA, Garganeeva AA, Aleksandrenko VA, Fedyunina VA, Ogurkova ON. Growth differentiation factor 15 associations with clinical features of chronic heart failure with midrange ejection fraction and preserved ejection fraction depending on the history of myocardial infarction. Kardiologiia. 2021;61(5):59-64.

18. Ardelean CL, Pescariu S, Lighezan DF, Pleava R, Ursoniu S, Nadasan V, Mihaicuta S. Particularities of older patients with obstructive sleep apnea and heart failure with mid-range ejection fraction. Medicina (Kaunas). 2019;55(8):449.

19. Doeblin P, Hashemi D, Tanacli R, Lapinskas T, Gebker R, Stehning C, Motzkus LA, et al. CMR Tissue Characterization in Patients with HFmrEF. J Clin Med. 2019;8(11):1877.

20. Senen K, Ileri M, Alper A, Yetkin F, Atak R, Hisar I, Yetkin E, et al. Increased levels of soluble adhesion molecules E-selectin and P-selectin in patients with cardiac syndrome X. Angiology. 2005;56(3):273-277.

21. Danilogorskaya Y, Belenkov YU, Privalova E, Zheleznykh E, Shakaryants G, Kozhevnikova M, Schendrygina A, et al. [PP.20.10] Endothelial dysfunction and vascular remodeling in patients with essential arterial hypertension. Journal of Hypertension. 2017;35:e25. 